\title{
Dynamics of a modified Nicholson-Bailey host-parasitoid model
}

\section{Abdul Qadeer Khan* and Muhammad Naeem Qureshi}

${ }^{\text {"Correspondence: }}$ abdulqadeerkhan1@gmail.com Department of Mathematics, University of Azad Jammu and Kashmir, Muzaffarabad, 13100, Pakistan

\section{Introduction}

Many ecological models are governed by differential as well as difference equations. In particular, ecological models with non-overlapping populations are better formulated as discrete dynamical systems compared to the continuous time models. These models have been extensively studied in recent years because of their wide applicability to the study of population dynamics [1, 2]. In fact, in the case of discrete dynamical systems, one has more efficient computational results for numerical simulations and also has rich dynamics as compared to the continuous ones. In recent years, several papers have been published on the mathematical models of biology that discuss the system of difference equations generated from the associated system of differential equations as well as the associated numerical methods [3, 4]. In mathematical biology, the model such as the host-parasitoid has attracted many researchers during the last few decades. Usually, the biologists believe that a unique, positive, locally asymptotically stable equilibrium point in an ecological system is very important [5]. Therefore, it is pertinent to find conditions which may guarantee the global stability of a positive equilibrium point, if it exist, for the given system. See [6] for introduction to mathematical models in biological sciences.

The prime example of an ecologically interesting discrete-time model for interacting populations is the Nicholson-Bailey model for host-parasitoid dynamics. Parasitoids are insect species whose larvae develop by feeding on the bodies of other arthropods, usually

(C) 2015 Khan and Qureshi; licensee Springer. This is an Open Access article distributed under the terms of the Creative Commons Attribution License (http://creativecommons.org/licenses/by/4.0), which permits unrestricted use, distribution, and reproduction in any medium, provided the original work is properly credited. 
killing them. Larvae emerge from the host and develop into free-living adults. The adults then lay their eggs in a subsequent generation of hosts. Most parasitoid larvae require a specific life-stage of the host, so parasitoid and host generations are linked to one another. Consequently host-parasitoid models often use a discrete time step corresponding to the common generation length of host and parasitoid. The classic model was derived by Nicholson and Bailey (1935). The assumptions of the Nicholson-Bailey model are as follows:

(i) Hosts are distributed at random, at density $x_{n}$ per unit area in generation $n$.

(ii) Parasitoids search at random and independently, each having an 'area of discovery' $a$, and lay an egg in each host found.

(iii) Each parasitized host gives rise to one new parasitoid in generation $n+1$.

(iv) Each unparasitized host gives rise to $b>1$ new hosts in generation $n+1$.

Each parasitoid attacks the hosts found in $a$ units of area, so the expected number of hosts attacked by each parasitoid is $a x$. The expected total number of attacks is $a x y$. The total number of attacks can also be written as the sum over hosts of the number of attacks on each host. All hosts have the same expected number of attacks, so the expected number of attacks on any given host must be ay. Under the assumptions listed above, the number of eggs per host has a Poisson distribution. Consequently, the expected fraction of hosts that are not parasitized is the probability that a Poisson random variable with mean ay takes the value zero. The resulting population dynamics are

$$
x_{n+1}=b x_{n} e^{-a y_{n}}, \quad y_{n+1}=c x_{n}\left(1-e^{-a y_{n}}\right) .
$$

Here, $x_{n}$ and $y_{n}$ represent the densities of the host and parasitoid population at year $n$. $b$ is the number of offspring of an unparasitized host surviving to the next year. Assuming random encounter between hosts and parasitoids, the probability that a host escapes parasitism can be approximated by $e^{-a y_{n}}$, where $a$ is a proportionality constant. Similarly, the probability to become infected is then given by $1-e^{-a y_{n}}$. The parameter $c$ describes the number of parasitoids that hatch from an infected host.

Now, assume that the host has bounded dynamics in absence of parasitoid, i.e., has selfregulation (density dependence). For example, assume host dynamics are inherently logistic (e.g., the Beverton-Holt model).

Then a modified form of the Nicholson-Bailey host-parasitoid model is

$$
x_{n+1}=\frac{b x_{n} e^{-a y_{n}}}{1+d x_{n}}, \quad y_{n+1}=c x_{n}\left(1-e^{-a y_{n}}\right),
$$

where $a, b, c, d$ and the initial conditions $x_{0}, y_{0}$ are positive real numbers.

In this paper our aim is to study the dynamics of system (1). More precisely, we investigate the boundedness character, existence and uniqueness of a positive equilibrium point, local asymptotic stability and global stability of the unique positive equilibrium point, and the rate of convergence of positive solutions of system (1). To investigate the dynamics we shall use standard results from theory of rational difference equations. However, we shall state the results that we employ and refer the interested readers for a systematic study of rational difference equations to [7-21] and the references therein. In Refs. [22-26] qualitative behavior of some biological models is discussed. The rest of the paper is organized as follows. In Section 2 the required known results about linearized stability are given. 
Section 3 discusses the boundedness character of the model. Section 4 is about the existence and uniqueness of the positive equilibrium point. It also contains the local stability of the equilibrium point. Section 5 discusses the global behavior of the equilibrium point. Whereas Section 6 is about the rate of convergence and Section 7 gives the numerical examples of the proved results. In the last section a brief conclusion is given.

\section{Linearized stability}

Let us consider the two-dimensional discrete dynamical system of the form

$$
\begin{aligned}
& x_{n+1}=f\left(x_{n}, y_{n}\right), \\
& y_{n+1}=g\left(x_{n}, y_{n}\right), \quad n=0,1, \ldots,
\end{aligned}
$$

where $f: I \times J \rightarrow I$ and $g: I \times J \rightarrow J$ are continuously differentiable functions and $I, J$ are some intervals of real numbers. Furthermore, a solution $\left\{\left(x_{n}, y_{n}\right)\right\}_{n=0}^{\infty}$ of system (2) is uniquely determined by initial conditions $\left(x_{0}, y_{0}\right) \in I \times J$. An equilibrium point of (2) is a point $(\bar{x}, \bar{y})$ that satisfies

$$
\begin{aligned}
& \bar{x}=f(\bar{x}, \bar{y}), \\
& \bar{y}=g(\bar{x}, \bar{y}) .
\end{aligned}
$$

Definition 2.1 Let $(\bar{x}, \bar{y})$ be an equilibrium point of system (2).

(i) An equilibrium point $(\bar{x}, \bar{y})$ is said to be stable if for every $\varepsilon>0$, there exists $\delta>0$ such that for every initial condition $\left(x_{0}, y_{0}\right),\left\|\left(x_{0}, y_{0}\right)-(\bar{x}, \bar{y})\right\|<\delta$ implies $\left\|\left(x_{n}, y_{n}\right)-(\bar{x}, \bar{y})\right\|<\varepsilon$ for all $n>0$, where $\|\cdot\|$ is the usual Euclidian norm in $\mathbb{R}^{2}$.

(ii) An equilibrium point $(\bar{x}, \bar{y})$ is said to be unstable if it is not stable.

(iii) An equilibrium point $(\bar{x}, \bar{y})$ is said to be asymptotically stable if there exists $\eta>0$ such that $\left\|\left(x_{0}, y_{0}\right)-(\bar{x}, \bar{y})\right\|<\eta$ and $\left(x_{n}, y_{n}\right) \rightarrow(\bar{x}, \bar{y})$ as $n \rightarrow \infty$.

(iv) An equilibrium point $(\bar{x}, \bar{y})$ is called a global attractor if $\left(x_{n}, y_{n}\right) \rightarrow(\bar{x}, \bar{y})$ as $n \rightarrow \infty$.

(v) An equilibrium point $(\bar{x}, \bar{y})$ is called an asymptotic global attractor if it is a global attractor and stable.

Definition 2.2 Let $(\bar{x}, \bar{y})$ be an equilibrium point of the map $F(x, y)=(f(x, y), g(x, y))$, where $f$ and $g$ are continuously differentiable functions at $(\bar{x}, \bar{y})$. The linearized system of (2) about the equilibrium point $(\bar{x}, \bar{y})$ is given by

$$
X_{n+1}=F\left(X_{n}\right)=F_{J} X_{n},
$$

where $X_{n}=\left(\begin{array}{l}x_{n} \\ y_{n}\end{array}\right)$ and $F_{J}$ is a Jacobian matrix of system (2) about the equilibrium point $(\bar{x}, \bar{y})$.

Let $(\bar{x}, \bar{y})$ be an equilibrium point of system (1), then

$$
\bar{x}=\frac{b \bar{x} e^{-a \bar{y}}}{1+d \bar{x}}, \quad \bar{y}=c \bar{x}\left(1-e^{-a \bar{y}}\right) .
$$

The Jacobian matrix of the linearized system of (1) about the fixed point $(\bar{x}, \bar{y})$ is given by

$$
F_{J}(\bar{x}, \bar{y})=\left(\begin{array}{cc}
\frac{b e^{-a \bar{y}}}{(1+d \bar{x})^{2}} & -\frac{a b \bar{x} e^{-a \bar{y}}}{1+d \bar{x}} \\
c\left(1-e^{-a \bar{y}}\right) & a c \bar{x} e^{-a \bar{y}}
\end{array}\right) .
$$


Lemma 2.3 [7] Consider the second-degree polynomial equation

$$
\lambda^{2}-p \lambda-q=0
$$

where $p$ and $q$ are real numbers.

(i) If both roots of Equation (4) lie in the open unit disk $|\lambda|<1$, then the equilibrium point $(\bar{x}, \bar{y})$ is locally asymptotically stable.

(ii) If at least one of the roots of Equation (4) has absolute value greater than one, then the equilibrium point $(\bar{x}, \bar{y})$ is unstable.

(iii) A necessary and sufficient condition for both roots of Equation (4) to lie inside the open disk $|\lambda|<1$ is

$$
|p|<1-q<2
$$

In this case the locally asymptotically stable equilibrium $(\bar{x}, \bar{y})$ is also called a sink.

(iv) A necessary and sufficient condition for both roots of Equation (4) to have absolute value greater than one is

$$
|q|>1, \quad|p|<|1-q| .
$$

In this case $(\bar{x}, \bar{y})$ is a repeller.

(v) A necessary and sufficient condition for one root of Equation (4) to have absolute value greater than one and for the other to have absolute value less than one is

$$
p^{2}+4 q>0, \quad|p|>|1-q| .
$$

In this case the unstable equilibrium $(\bar{x}, \bar{y})$ is called a saddle point.

(vi) A necessary and sufficient condition for a root of Equation (4) to have absolute value equal to one is

$$
|p|=|1-q|
$$

In this case the equilibrium $(\bar{x}, \bar{y})$ is called a non-hyperbolic point.

\section{Boundedness}

The following theorem shows that every positive solution $\left\{\left(x_{n}, y_{n}\right)\right\}_{n=0}^{\infty}$ of system (1) is bounded.

Theorem 3.1 Every positive solution $\left\{\left(x_{n}, y_{n}\right)\right\}_{n=0}^{\infty}$ of system (1) is bounded.

Proof Let $\left\{\left(x_{n}, x_{n}\right)\right\}_{n=0}^{\infty}$ be any positive solution of system (1), then

$$
x_{n+1}=\frac{b x_{n} e^{-a y_{n}}}{1+d x_{n}} \leq \frac{b x_{n}}{d x_{n}} \leq \frac{b}{d}, \quad n=0,1, \ldots
$$

Also

$$
y_{n+1}=c x_{n}\left(1-e^{-a y_{n}}\right) \leq c x_{n} \leq \frac{b c}{d}, \quad n=0,1, \ldots
$$


Hence from (5) and (6), we have

$$
0 \leq x_{n} \leq \frac{b}{d}, \quad 0 \leq y_{n} \leq \frac{b c}{d}, \quad n=1,2, \ldots
$$

Theorem 3.2 Let $\left\{\left(x_{n}, y_{n}\right)\right\}$ be a positive solution of system (1). Then $\left[0, \frac{b}{d}\right] \times\left[0, \frac{b c}{d}\right]$ is an invariant set for system (1).

Proof It follows from induction.

\section{Existence and uniqueness of a positive equilibrium point and local stability}

The following theorem shows the existence and uniqueness of a positive equilibrium point of system (1).

Theorem 4.1 If $b>1$ and $d<\frac{a c}{b \ln \left(\frac{1+b}{b}\right)}$, then system (1) has a unique positive equilibrium point $(\bar{x}, \bar{y})$ in $\left[0, \frac{b}{d}\right] \times\left[0, \frac{b c}{d}\right]$.

Proof Consider the following system:

$$
x=\frac{b x e^{-a y}}{1+d x}, \quad y=c x\left(1-e^{-a y}\right) .
$$

Assume that $(\bar{x}, \bar{y})$ in $\left[0, \frac{b}{d}\right] \times\left[0, \frac{b c}{d}\right]$, then it follows from (8) that

$$
y=\frac{1}{a} \ln \left(\frac{b}{1+d x}\right), \quad x=\frac{y}{c\left(1-e^{-a y}\right)} .
$$

Define $F(x)=\frac{h(x)}{c\left(1-e^{-a h(x)}\right)}-x$, where $h(x)=\frac{1}{a} \ln \left(\frac{b}{1+d x}\right)$ and $x \in\left[0, \frac{b}{d}\right]$. It is easy to see that $F(0)=\frac{b \ln b}{a c(b-1)}>0$ if $b>1$. Also, $F\left(\frac{b}{d}\right)=\frac{b \ln \left(\frac{1+b}{b}\right)}{a c}-\frac{b}{d}<0$ if $d<\frac{a c}{b \ln \left(\frac{1+b}{b}\right)}$. Hence, $F(x)$ has at least one positive solution in the interval $\left[0, \frac{b}{d}\right]$. Furthermore, it is easy to show that $F^{\prime}(x)=$ $\frac{\left(1-e^{-a h(x)}-a h(x) e^{-a h(x)}\right) h^{\prime}(x)-c\left(1-e^{-a h(x)}\right)^{2}}{c\left(1-e^{-a h(x)}\right)^{2}}<0$, where $h^{\prime}(x)=-\frac{d}{a(1+d x)}$ for all $x \in\left[0, \frac{b}{d}\right]$. Hence, $F(x)=$ 0 has a unique positive solution $x \in\left[0, \frac{b}{d}\right]$.

Theorem 4.2 For the unique positive equilibrium point $(\bar{x}, \bar{y})$ in $\left[0, \frac{b}{d}\right] \times\left[0, \frac{b c}{d}\right]$ of system (1), the following statements hold true:

(i) The unique positive equilibrium point of system (1) is locally asymptotically stable if and only if

$$
\begin{aligned}
& \frac{b e^{a c r(b d r+1-b)}\left(a c r(1+b d r)^{2}+1\right)}{(1+b d r)^{2}} \\
& <1-\frac{a b^{2} c r e^{a c r(b d r+1-b)}\left(b d r\left(e^{a c r(b d r+1-b)}-1\right)-1\right)}{(1+b d r)^{2}}<2 .
\end{aligned}
$$

(ii) The unique positive equilibrium point is a repeller if and only if

$$
\left|\frac{a b^{2} c r e^{a c r(b d r+1-b)}\left(b d r\left(e^{a c r(b d r+1-b)}-1\right)-1\right)}{(1+b d r)^{2}}\right|>1
$$


and

$$
\begin{aligned}
& \frac{b e^{a c r(b d r+1-b)}\left(a c r(1+b d r)^{2}+1\right)}{(1+b d r)^{2}} \\
& <\left|1-\frac{a b^{2} c r e^{a c r(b d r+1-b)}\left(b d r\left(e^{a c r(b d r+1-b)}-1\right)-1\right)}{(1+b d r)^{2}}\right| .
\end{aligned}
$$

(iii) The unique positive equilibrium point is a saddle point if and only if

$$
\begin{aligned}
& \left(\frac{b e^{a c r(b d r+1-b)}\left(a c r(1+b d r)^{2}+1\right)}{(1+b d r)^{2}}\right)^{2} \\
& +4\left(\frac{a b^{2} c r e^{a c r(b d r+1-b)}\left(b d r\left(e^{a c r(b d r+1-b)}-1\right)-1\right)}{(1+b d r)^{2}}\right)>0
\end{aligned}
$$

and

$$
\begin{aligned}
& \frac{b e^{a c r(b d r+1-b)}\left(a c r(1+b d r)^{2}+1\right)}{(1+b d r)^{2}} \\
& >\left|1-\frac{a b^{2} c r e^{a c r(b d r+1-b)}\left(b d r\left(e^{a c r(b d r+1-b)}-1\right)-1\right)}{(1+b d r)^{2}}\right|
\end{aligned}
$$

(iv) The unique positive equilibrium point is non-hyperbolic if and only if

$$
\begin{aligned}
& \frac{b e^{a c r(b d r+1-b)}\left(a c r(1+b d r)^{2}+1\right)}{(1+b d r)^{2}} \\
& \quad=\left|1-\frac{a b^{2} c r e^{a c r(b d r+1-b)}\left(b d r\left(e^{a c r(b d r+1-b)}-1\right)-1\right)}{(1+b d r)^{2}}\right| .
\end{aligned}
$$

Proof (i) The characteristic polynomial of the Jacobian matrix $F_{J}(\bar{x}, \bar{y})$ about the equilibrium point $(\bar{x}, \bar{y})$ is given by

$$
P(\lambda)=\lambda^{2}-\frac{e^{-a \bar{y}}\left(a c \bar{x}(1+d \bar{x})^{2}+b\right)}{(1+d \bar{x})^{2}} \lambda+\frac{a b c \bar{x} e^{-2 a \bar{y}}\left(e^{a \bar{y}}(1+d \bar{x})-d \bar{x}\right)}{(1+d \bar{x})^{2}} .
$$

As pointed out in [27], it is convenient to discuss stability behavior in terms of the quantity $r$. So, for the equilibrium point $(\bar{x}, \bar{y})$ of system (1), we have from system (3)

$$
e^{-a \bar{y}}=\frac{1}{b}+d r
$$

where $r=\frac{\bar{x}}{b}$ is the ratio of steady-state $\bar{x}$ with $b$. Moreover,

$$
\bar{y}=c r(b-1-b d r) .
$$

So, in terms of $r$, Equation (9) takes the form

$$
\begin{aligned}
P(\lambda)= & \lambda^{2}-\frac{b e^{a c r(b d r+1-b)}\left(a c r(1+b d r)^{2}+1\right)}{(1+b d r)^{2}} \lambda \\
& -\frac{a b^{2} c r e^{a c r(b d r+1-b)}\left(b d r\left(e^{a c r(b d r+1-b)}-1\right)-1\right)}{(1+b d r)^{2}} .
\end{aligned}
$$


Let

$$
\begin{aligned}
& p=\frac{b e^{a c r(b d r+1-b)}\left(a c r(1+b d r)^{2}+1\right)}{(1+b d r)^{2}} \\
& q=\frac{a b^{2} c r e^{a c r(b d r+1-b)}\left(b d r\left(e^{a c r(b d r+1-b)}-1\right)-1\right)}{(1+b d r)^{2}}
\end{aligned}
$$

Then it follows from Lemma 2.3 that the unique positive equilibrium point $(\bar{x}, \bar{y})$ of system (1) is locally asymptotically stable if and only if

$$
\frac{b e^{a c r(b d r+1-b)}\left(a c r(1+b d r)^{2}+1\right)}{(1+b d r)^{2}}<1-\frac{a b^{2} c r e^{a c r(b d r+1-b)}\left(b d r\left(e^{a c r(b d r+1-b)}-1\right)-1\right)}{(1+b d r)^{2}}<2 .
$$

Obviously, one can prove (ii), (iii) and (iv).

\section{Global character}

Lemma 5.1 [7] Let $I=[a, b]$ and $J=[c, d]$ be real intervals, and let $f: I \times J \rightarrow I$ and $g: I \times$ $J \rightarrow J$ be continuous functions. Consider system (2) with initial conditions $\left(x_{0}, y_{0}\right) \in I \times J$. Suppose that the following statements are true:

(i) $f(x, y)$ is non-decreasing in $x$ and non-increasing in $y$.

(ii) $g(x, y)$ is non-decreasing in both arguments.

(iii) If $\left(m_{1}, M_{1}, m_{2}, M_{2}\right) \in I^{2} \times J^{2}$ is a solution of the system

$$
\begin{array}{ll}
m_{1}=f\left(m_{1}, M_{2}\right), & M_{1}=f\left(M_{1}, m_{2}\right), \\
m_{2}=g\left(m_{1}, m_{2}\right), & M_{2}=g\left(M_{1}, M_{2}\right)
\end{array}
$$

such that $m_{1}=M_{1}$ and $m_{2}=M_{2}$, then there exists exactly one equilibrium point $(\bar{x}, \bar{y})$ of system (2) such that $\lim _{n \rightarrow \infty}\left(x_{n}, y_{n}\right)=(\bar{x}, \bar{y})$.

Theorem 5.2 Assume that $a c+d>a b c$, then the unique positive equilibrium point $(\bar{x}, \bar{y})$ in $\left[0, \frac{b}{d}\right] \times\left[0, \frac{b c}{d}\right]$ of system (1) is a global attractor.

Proof Let $f(x, y)=\frac{b x e^{-a y}}{1+d x}$ and $g(x, y)=c x\left(1-e^{-a y}\right)$. Then it is easy to see that $f(x, y)$ is nondecreasing in $x$ and non-increasing in $y$. Moreover, $g(x, y)$ is non-decreasing in both arguments $x$ and $y$. Let $\left(m_{1}, M_{1}, m_{2}, M_{2}\right)$ be a positive solution of the system

$$
\begin{array}{ll}
m_{1}=f\left(m_{1}, M_{2}\right), & M_{1}=f\left(M_{1}, m_{2}\right), \\
m_{2}=g\left(m_{1}, m_{2}\right), & M_{2}=g\left(M_{1}, M_{2}\right) .
\end{array}
$$

Then one has

$$
m_{1}=\frac{b m_{1} e^{-a M_{2}}}{1+d m_{1}}, \quad M_{1}=\frac{b M_{1} e^{-a m_{2}}}{1+d M_{1}}
$$

and

$$
m_{2}=c m_{1}\left(1-e^{-a m_{2}}\right), \quad M_{2}=c M_{1}\left(1-e^{-a M_{2}}\right) .
$$


From (10) one has

$$
m_{1}=\frac{b e^{-a M_{2}}-1}{d}, \quad M_{1}=\frac{b e^{-a m_{2}}-1}{d} .
$$

From (11) one has

$$
m_{2}=\frac{c\left(b e^{-a M_{2}}-1\right)\left(1-e^{-a m_{2}}\right)}{d}, \quad M_{2}=\frac{c\left(b e^{-a m_{2}}-1\right)\left(1-e^{-a M_{2}}\right)}{d} .
$$

From (12) we have

$$
m_{1}-M_{1}=\frac{b}{d} e^{-a m_{2}-a M_{2}}\left(e^{a m_{2}}-e^{a M_{2}}\right) .
$$

From (13) we have

$$
m_{2}-M_{2}=\frac{c(b-1)}{d} e^{-a m_{2}-a M_{2}}\left(e^{a m_{2}}-e^{a M_{2}}\right) .
$$

Moreover, one has

$$
e^{a m_{2}}-e^{a M_{2}}=a e^{\gamma}\left(m_{2}-M_{2}\right),
$$

where

$$
\min \left\{m_{2}, M_{2}\right\} \leq \gamma \leq \max \left\{m_{2}, M_{2}\right\} .
$$

From (14) and (16), we get

$$
m_{1}-M_{1}=\frac{a b}{d} e^{-a m_{2}-a M_{2}+\gamma}\left(m_{2}-M_{2}\right) .
$$

From (17) it follows that

$$
\left|m_{1}-M_{1}\right| \leq \frac{a b}{d}\left|m_{2}-M_{2}\right|
$$

From (15) and (16), we get

$$
m_{2}-M_{2}=\frac{a c(b-1)}{d} e^{-a m_{2}-a M_{2}+\gamma}\left(m_{2}-M_{2}\right) .
$$

From (19) it follows that

$$
\left|m_{2}-M_{2}\right| \leq \frac{a c(b-1)}{d}\left|m_{2}-M_{2}\right|
$$

From (20) we get

$$
\left(\frac{a c+d-a b c}{d}\right)\left|m_{2}-M_{2}\right| \leq 0,
$$

from which it follows that $m_{2}=M_{2}$ and similarly it is easy to show that $m_{1}=M_{1}$. Hence, from Lemma 5.1 the unique positive equilibrium point of system (1) is a global attractor. 
Lemma 5.3 The unique positive equilibrium point $(\bar{x}, \bar{y})$ in $\left[0, \frac{b}{d}\right] \times\left[0, \frac{b c}{d}\right]$ of system (1) is globally asymptotically stable if and only if

$$
\frac{b e^{a c r(b d r+1-b)}\left(a c r(1+b d r)^{2}+1\right)}{(1+b d r)^{2}}<1-\frac{a b^{2} c r e e^{a c r(b d r+1-b)}\left(b d r\left(e^{a c r(b d r+1-b)}-1\right)-1\right)}{(1+b d r)^{2}}<2 .
$$

Proof The proof is a direct consequence of Theorems 4.2 and 5.2.

\section{The rate of convergence}

In this section we will determine the rate of convergence of a solution that converges to the unique positive equilibrium point of system (1).

The following result gives the rate of convergence of solutions of the system of difference equations

$$
X_{n+1}=(A+B(n)) X_{n}
$$

where $X_{n}$ is an $m$-dimensional vector, $A \in C^{m \times m}$ is a constant matrix, and $B: \mathbb{Z}^{+} \rightarrow C^{m \times m}$ is a matrix function satisfying

$$
\|B(n)\| \rightarrow 0
$$

as $n \rightarrow \infty$, where $\|\cdot\|$ denotes any matrix norm which is associated with the vector norm

$$
\|(x, y)\|=\sqrt{x^{2}+y^{2}} .
$$

Proposition 6.1 (Perron's theorem [28]) Suppose that condition (22) holds. If $X_{n}$ is a solution of (21), then either $X_{n}=0$ for all large $n$ or

$$
\rho=\lim _{n \rightarrow \infty}\left(\left\|X_{n}\right\|\right)^{1 / n}
$$

exists and is equal to the modulus of one of the eigenvalues of matrix $A$.

Proposition 6.2 [28] Suppose that condition (22) holds. If $X_{n}$ is a solution of (21), then either $X_{n}=0$ for all large $n$ or

$$
\rho=\lim _{n \rightarrow \infty} \frac{\left\|X_{n+1}\right\|}{\left\|X_{n}\right\|}
$$

exists and is equal to the modulus of one of the eigenvalues of matrix $A$.

Let $\left\{\left(x_{n}, y_{n}\right)\right\}$ be any solution of system (1) such that $\lim _{n \rightarrow \infty} x_{n}=\bar{x}$ and $\lim _{n \rightarrow \infty} y_{n}=\bar{y}$. To find the error terms, one has from system (1)

$$
\begin{aligned}
x_{n+1}-\bar{x} & =\frac{b x_{n} e^{-a y_{n}}}{1+d x_{n}}-\frac{b \bar{x} e^{-a \bar{y}}}{1+d \bar{x}} \\
& =\frac{b e^{-a y_{n}}}{\left(1+d x_{n}\right)(1+d \bar{x})}\left(x_{n}-\bar{x}\right)+\frac{b \bar{x}\left(e^{-a y_{n}}-e^{-a \bar{y}}\right)}{(1+d \bar{x})\left(y_{n}-\bar{y}\right)}\left(y_{n}-\bar{y}\right)
\end{aligned}
$$


and

$$
\begin{aligned}
y_{n+1}-\bar{y} & =c x_{n}\left(1-e^{-a y_{n}}\right)-c \bar{x}\left(1-e^{-a \bar{y}}\right) \\
& =c\left(1-e^{-a y_{n}}\right)\left(x_{n}-\bar{x}\right)-\frac{c \bar{x}\left(e^{-a y_{n}}-e^{-a \bar{y}}\right)}{y_{n}-\bar{y}}\left(y_{n}-\bar{y}\right) .
\end{aligned}
$$

Let $e_{n}^{1}=x_{n}-\bar{x}$ and $e_{n}^{2}=y_{n}-\bar{y}$, then one has

$$
e_{n+1}^{1}=a_{n} e_{n}^{1}+b_{n} e_{n}^{2}
$$

and

$$
e_{n+1}^{2}=c_{n} e_{n}^{1}+d_{n} e_{n}^{2}
$$

where

$$
\begin{aligned}
& a_{n}=\frac{b e^{-a y_{n}}}{\left(1+d x_{n}\right)(1+d \bar{x})}, \quad b_{n}=\frac{b \bar{x}\left(e^{-a y_{n}}-e^{-a \bar{y}}\right)}{(1+d \bar{x})\left(y_{n}-\bar{y}\right)}, \\
& c_{n}=c\left(1-e^{-a y_{n}}\right), \quad d_{n}=-\frac{c \bar{x}\left(e^{-a y_{n}}-e^{-a \bar{y}}\right)}{y_{n}-\bar{y}} .
\end{aligned}
$$

Moreover,

$$
\begin{array}{ll}
\lim _{n \rightarrow \infty} a_{n}=\frac{b e^{-a \bar{y}}}{(1+d \bar{x})^{2}}, & \lim _{n \rightarrow \infty} b_{n}=-\frac{a b \bar{x} e^{-a \bar{y}}}{1+d \bar{x}}, \\
\lim _{n \rightarrow \infty} c_{n}=c\left(1-e^{-a \bar{y}}\right), & \lim _{n \rightarrow \infty} d_{n}=a c \bar{x} e^{-a \bar{y}} .
\end{array}
$$

Now the limiting system of error terms can be written as

$$
\left(\begin{array}{c}
e_{n+1}^{1} \\
e_{n+1}^{2}
\end{array}\right)=\left(\begin{array}{cc}
\frac{b e^{-a \bar{y}}}{(1+d \bar{x})^{2}} & -\frac{a b \bar{x} e^{-a \bar{y}}}{1+d \bar{x}} \\
c\left(1-e^{-a \bar{y}}\right) & a c \bar{x} e^{-a \bar{y}}
\end{array}\right)\left(\begin{array}{l}
e_{n}^{1} \\
e_{n}^{2}
\end{array}\right)
$$

which is similar to the linearized system of (1) about the equilibrium point $(\bar{x}, \bar{y})$.

Using proposition (6.1), one has following result.

Theorem 6.3 Assume that $\left\{\left(x_{n}, y_{n}\right)\right\}$ is a positive solution of system (1) such that $\lim _{n \rightarrow \infty} x_{n}=\bar{x}$, and $\lim _{n \rightarrow \infty} y_{n}=\bar{y}$, where $\bar{x}$ in $\left[0, \frac{b}{d}\right]$ and $\bar{y}$ in $\left[0, \frac{b c}{d}\right]$. Then the error vector $e_{n}=\left(\begin{array}{c}e_{n}^{1} \\ e_{n}^{2}\end{array}\right)$ of every solution of (1) satisfies both of the following asymptotic relations:

$$
\lim _{n \rightarrow \infty}\left(\left\|e_{n}\right\|\right)^{\frac{1}{n}}=\left|\lambda_{1,2} F_{J}(\bar{x}, \bar{y})\right|, \quad \lim _{n \rightarrow \infty} \frac{\left\|e_{n+1}\right\|}{\left\|e_{n}\right\|}=\left|\lambda_{1,2} F_{J}(\bar{x}, \bar{y})\right|,
$$

where $\lambda_{1,2} F_{J}(\bar{x}, \bar{y})$ are the characteristic roots of the Jacobian matrix $F_{J}(\bar{x}, \bar{y})$.

\section{Examples}

In order to support our theoretical discussions, we consider several interesting numerical examples in this section. These examples represent different types of qualitative behavior of solutions to the system of nonlinear difference equations (1). All plots in this section are drawn with Mathematica. 


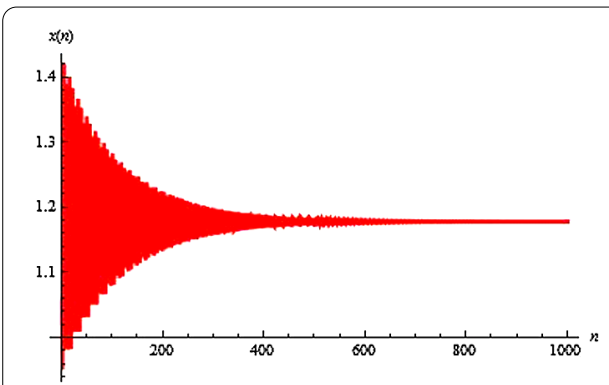

(a) Plot of $x_{n}$ for system (23)

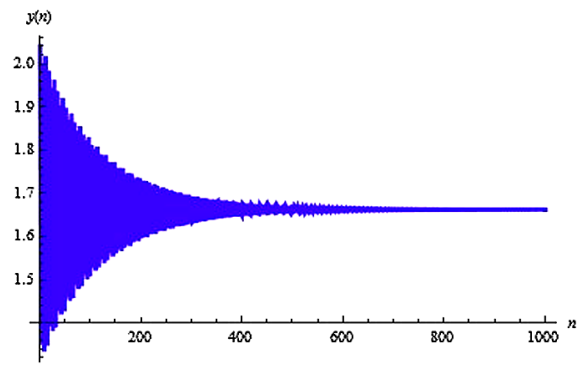

(b) Plot of $y_{n}$ for system (23)

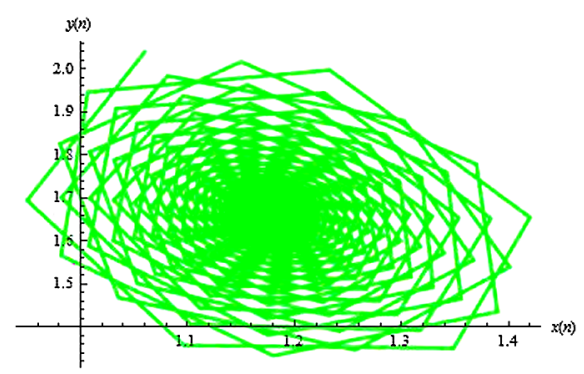

(c) An attractor of system (23)

Figure 1 Plots for system (23).

Example 1 Let $a=0.5, b=9.6, c=2.5, d=2.7$. Then system (1) can be written as

$$
x_{n+1}=\frac{9.6 x_{n} e^{-0.5 y_{n}}}{1+2.7 x_{n}}, \quad y_{n+1}=2.5 x_{n}\left(1-e^{-0.5 y_{n}}\right), \quad n=0,1, \ldots
$$

with initial conditions $x_{0}=1.06, y_{0}=2.04$.

In this case the unique positive equilibrium point of system $(23)$ is given by $(\bar{x}, \bar{y})=$ $(1.17805,1.66255)$. Moreover, in Figure 1 the plot of $x_{n}$ is shown in Figure 1(a), the plot of $y_{n}$ is shown in Figure 1(b) and an attractor of system (23) is shown in Figure 1(c).

Example 2 Let $a=0.4, b=8.7, c=5.5, d=4.7$. Then system (1) can be written as

$$
x_{n+1}=\frac{8.7 x_{n} e^{-0.4 y_{n}}}{1+4.7 x_{n}}, \quad y_{n+1}=5.5 x_{n}\left(1-e^{-0.4 y_{n}}\right), \quad n=0,1, \ldots
$$

with initial conditions $x_{0}=0.27, y_{0}=2.04$.

In this case the unique positive equilibrium point of system $(24)$ is given by $(\bar{x}, \bar{y})=$ $(0.649926,1.90865)$. Moreover, in Figure 2 the plot of $x_{n}$ is shown in Figure 2(a), the plot of $y_{n}$ is shown in Figure 2(b) and an attractor of system (24) is shown in Figure 2(c).

Example 3 Let $a=0.4, b=8.8, c=4.5, d=4.8$. Then system (1) can be written as

$$
x_{n+1}=\frac{8.8 x_{n} e^{-0.4 y_{n}}}{1+4.8 x_{n}}, \quad y_{n+1}=4.5 x_{n}\left(1-e^{-0.4 y_{n}}\right), \quad n=0,1, \ldots
$$

with initial conditions $x_{0}=0.28, y_{0}=2.04$. 


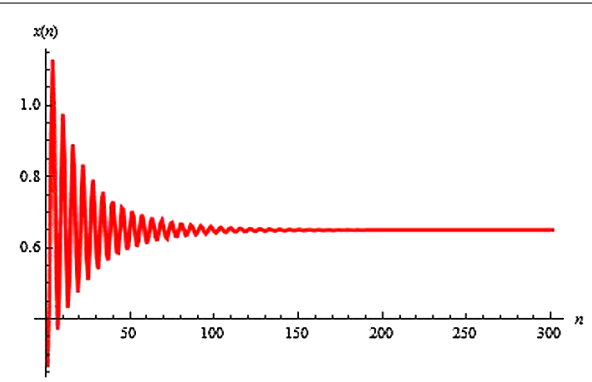

(a) Plot of $x_{n}$ for system (24)

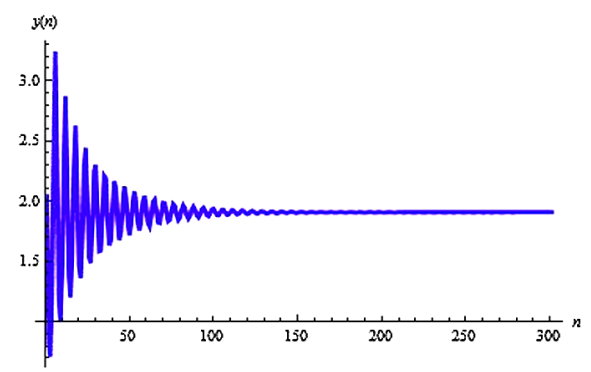

(b) Plot of $y_{n}$ for system (24)

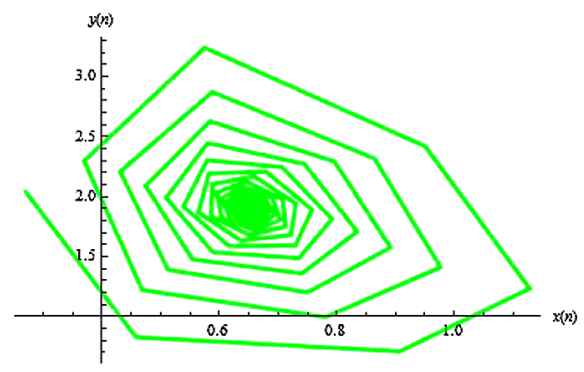

(c) An attractor of system (24)

Figure 2 Plots for system (24).

In this case the unique positive equilibrium point of system (25) is given by $(\bar{x}, \bar{y})=$ $(0.753772,1.61192)$. Moreover, in Figure 3 the plot of $x_{n}$ is shown in Figure 3(a), the plot of $y_{n}$ is shown in Figure 3(b) and an attractor of system (25) is shown in Figure $3(\mathrm{c})$.

Example 4 Let $a=0.4, b=8.2, c=2.4, d=1.7$. Then system (1) can be written as

$$
x_{n+1}=\frac{8.2 x_{n} e^{-0.4 y_{n}}}{1+1.7 x_{n}}, \quad y_{n+1}=2.4 x_{n}\left(1-e^{-0.4 y_{n}}\right), \quad n=0,1, \ldots
$$

with initial conditions $x_{0}=1.5, y_{0}=2.09$.

In this case the unique positive equilibrium point of system (26) is unstable. Moreover, in Figure 4 the plot of $x_{n}$ is shown in Figure 4(a), the plot of $y_{n}$ is shown in Figure 4(b) and a phase portrait of system (26) is shown in Figure 4(c).

Example 5 Let $a=0.4, b=8.6, c=3.9, d=2.9$. Then system (1) can be written as

$$
x_{n+1}=\frac{8.6 x_{n} e^{-0.4 y_{n}}}{1+2.9 x_{n}}, \quad y_{n+1}=3.9 x_{n}\left(1-e^{-0.4 y_{n}}\right), \quad n=0,1, \ldots
$$

with initial conditions $x_{0}=0.4, y_{0}=2.09$.

In this case the unique positive equilibrium point of system (27) is unstable. Moreover, in Figure 5 the plot of $x_{n}$ is shown in Figure 5(a), the plot of $y_{n}$ is shown in Figure 5(b) and a phase portrait of system (27) is shown in Figure 5(c). 


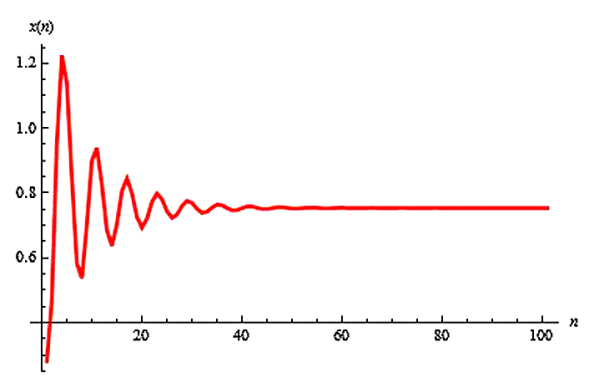

(a) Plot of $x_{n}$ for system (25)

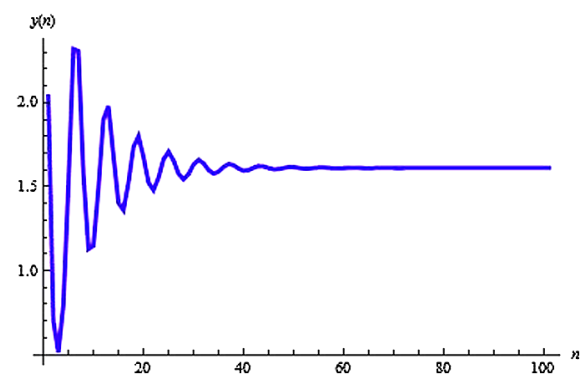

(b) Plot of $y_{n}$ for system (25)

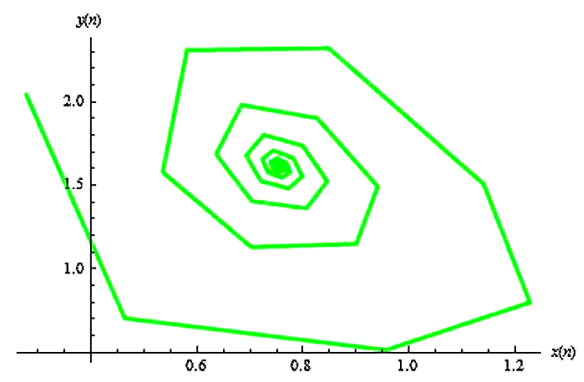

(c) An attractor of system (25)

Figure 3 Plots for system (25).

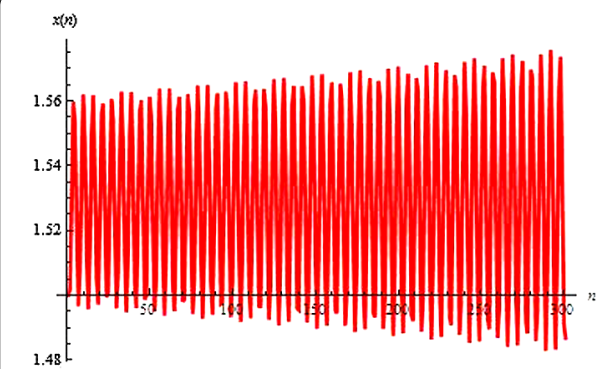

(a) Plot of $x_{n}$ for system (26)

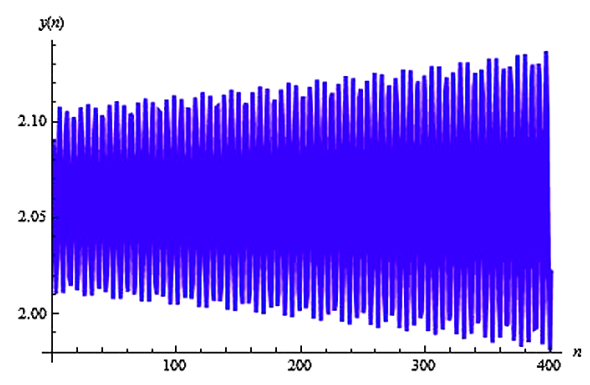

(b) Plot of $y_{n}$ for system (26)

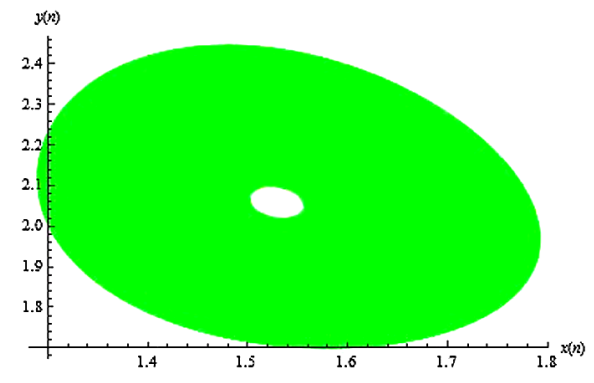

(c) Phase portrait of system (26)

Figure 4 Plots for system (26). 


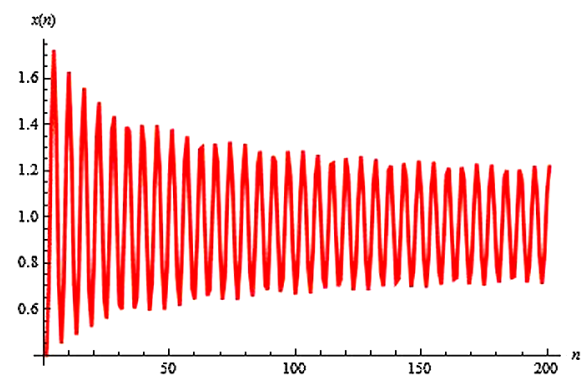

(a) Plot of $x_{n}$ for system (27)

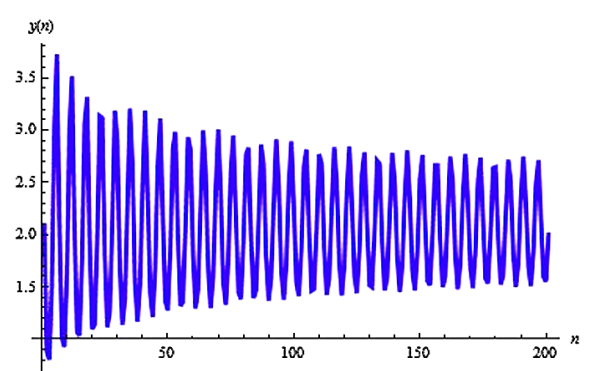

(b) Plot of $y_{n}$ for system (27)

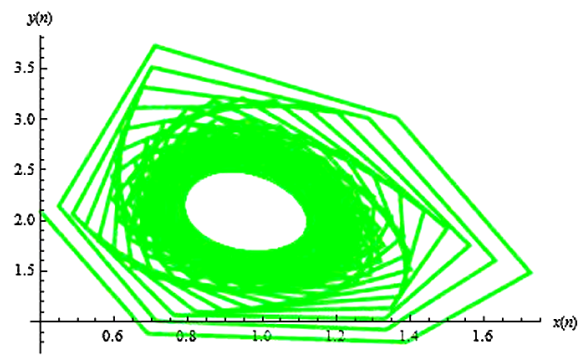

(c) Phase portrait of system (27)

Figure 5 Plots for system (27).

\section{Conclusion}

This work is related to the qualitative behavior of the modified Nicholson-Bailey hostparasitoid model. We have investigated the existence and uniqueness of positive steadystate of system (1). Under certain parametric conditions, the boundedness of positive solutions is proved. Moreover, we have shown that the unique positive equilibrium $(\bar{x}, \bar{y})$ in the $\left[0, \frac{b}{d}\right] \times\left[0, \frac{b c}{d}\right]$ point of system (1) is locally asymptotically stable if and only if $\frac{b e^{a c r(b d r+1-b)}\left(a c r(1+b d r)^{2}+1\right)}{(1+b d r)^{2}}<1-\frac{a b^{2} c r e e^{a c r(b d r+1-b)}\left(b d r\left(e^{a c r(b d r+1-b)}-1\right)-1\right)}{(1+b d r)^{2}}<2$ hold true. The main objective of dynamical systems theory is to predict the global behavior of a system based on the knowledge of its present state. An approach to this problem consists of determining possible global behaviors of the system and determining which initial conditions lead to these long-term behaviors. Furthermore, the rate of convergence of positive solutions of (1) which converge to its unique positive equilibrium point is demonstrated. Finally, some numerical examples are provided to support our theoretical results. These examples are experimental verification of our theoretical discussions.

Competing interests

The authors declare that they have no competing interests.

Authors' contributions

All authors contributed equally to the writing of this paper. All authors read and approved the final manuscript.

\section{Acknowledgements}

The authors thank the main editor and anonymous referees for their valuable comments and suggestions that led to the improvement of this paper. This work was supported by the Higher Education Commission of Pakistan.

Received: 3 October 2014 Accepted: 2 January 2015 Published online: 30 January 2015

References

1. Ahmad, S: On the nonautonomous Lotka-Volterra competition equation. Proc. Am. Math. Soc. 117, 199-204 (1993) 
2. Tang, X, Zou, X: On positive periodic solutions of Lotka-Volterra competition systems with deviating arguments. Proc. Am. Math. Soc. 134, 2967-2974 (2006)

3. Zhou, Z, Zou, X: Stable periodic solutions in a discrete periodic logistic equation. Appl. Math. Lett. 16(2), 165-171 (2003)

4. Liu, X: A note on the existence of periodic solution in discrete predator-prey models. Appl. Math. Model. 34, 2477-2483 (2010)

5. Kuang, Y: Global stability of Gause-type predator-prey systems. J. Math. Biol. 28, 463-474 (1990)

6. Allen, LJS: An Introduction to Mathematical Biology. Prentice Hall, New York (2007)

7. Grove, EA, Ladas, G: Periodicities in Nonlinear Difference Equations. Chapman \& Hall/CRC Press, Boca Raton (2004)

8. Sedaghat, H: Nonlinear Difference Equations: Theory with Applications to Social Science Models. Kluwer Academic, Dordrecht (2003)

9. Kocic, VL, Ladas, G: Global Behavior of Nonlinear Difference Equations of Higher Order with Applications. Kluwer Academic, Dordrecht (1993)

10. Khan, AQ, Qureshi, MN: Behavior of an exponential system of difference equations. Discrete Dyn. Nat. Soc. 2014 Article ID 607281 (2014). doi:10.1155/2014/607281

11. Khan, AQ: Global dynamics of two systems of exponential difference equations by Lyapunov function. Adv. Differ. Equ. 2014, 297 (2014)

12. Khan, $\mathrm{AQ}$, Qureshi, MN: Global dynamics of a competitive system of rational difference equations. Math. Methods Appl. Sci. (2014). doi:10.1002/mma.3392

13. Camouzis, E, Ladas, G: Dynamics of Third-Order Rational Difference Equations: With Open Problems and Conjectures. Chapman \& Hall/CRC Press, Boca Raton (2007)

14. Din, Q, Qureshi, MN, Khan, AQ: Dynamics of a fourth-order system of rational difference equations. Adv. Differ. Equ. 2012, $215(2012)$

15. Khan, AQ, Qureshi, MN, Din, Q: Global dynamics of some systems of higher-order rational difference equations. Adv. Differ. Equ. 2013, 354 (2013)

16. Shojaei, M, Saadati, R, Adibi, H: Stability and periodic character of a rational third order difference equation. Chaos Solitons Fractals 39, 1203-1209 (2009)

17. Kalabušić, S, Kulenović, MRS, Pilav, E: Global dynamics of a competitive system of rational difference equations in the plane. Adv. Differ. Equ. 2009, Article ID 132802 (2009)

18. Kalabušić, S, Kulenović, MRS, Pilav, E: Multiple attractors for a competitive system of rational difference equations in the plane. Abstr. Appl. Anal. (2011). doi:10.1155/2011/295308

19. Elsayed, EM: Behavior and expression of the solutions of some rational difference equations. J. Comput. Anal. Appl. 15(1), 73-81 (2013)

20. Elsayed, EM, El-Metwally, H: Stability and solutions for rational recursive sequence of order three. J. Comput. Anal. Appl. 17(2), 305-315 (2014)

21. Elsayed, EM, El-Metwally, HA: On the solutions of some nonlinear systems of difference equations. Adv. Differ. Equ. 2013, 16 (2013)

22. Qureshi, MN, Khan, AQ, Din, Q: Asymptotic behavior of a Nicholson-Bailey model. Adv. Differ. Equ. 2014, 62 (2014)

23. Ufuktepe, Ü, Kapçak, S: Stability analysis of a host parasite model. Adv. Differ. Equ. (2013). doi:10.1186/1687-1847-2013-79

24. Selgrade, JF, Ziehe, M: Convergence to equilibrium in a genetic model with differential viability between the sexes. J. Math. Biol. 25, 477-490 (1887)

25. Kalabušić, S, Kulenović, MRS, Pilav, E: Dynamics of a two-dimensional system of rational difference equations of Leslie-Gower type. Adv. Differ. Equ. (2011). doi:10.1186/1687-1847-2011-29

26. Din, Q, Khan, AQ, Qureshi, MN: Qualitative behavior of a host-pathogen model. Adv. Differ. Equ. (2013). doi:10.1186/1687-1847-2013-263

27. Edelstein-Keshet, L: Mathematical Models in Biology. McGraw-Hill, New York (1988)

28. Pituk, M: More on Poincare's and Perron's theorems for difference equations. J. Differ. Equ. Appl. 8, 201-216 (2002)

\section{Submit your manuscript to a SpringerOpen ${ }^{\ominus}$ journal and benefit from:}

- Convenient online submission

Rigorous peer review

- Immediate publication on acceptance

- Open access: articles freely available online

- High visibility within the field

- Retaining the copyright to your article 